\title{
Automated Bone Segmentation from Pelvic CT Images
}

\author{
Simina Vasilache and Kayvan Najarian \\ Virginia Commonwealth University \\ Department of Computer Science \\ Richmond, VA, 23284, USA \\ vasilaches@vcu.edu,knajarian@vcu.edu
}

\begin{abstract}
Segmentation of bone tissue from Pelvic CT images is a crucial step in developing an automated system for assisting experts with diagnostic decisions for traumatic pelvic injuries. The method proposed in this paper combines Wavelet processing, Laplacian filtering, morphology operations, a series of region growing techniques and gradient based segmentation methods to create an automated segmentation system. The method, tested against a database of pelvic injury CT images, provides promising results. This computationally efficient method sets the grounds for creating an automated decision making system that will be able to provide physicians with reliable recommendations for the treatment of traumatic pelvic injuries.
\end{abstract}

\section{Introduction}

Pelvic injury is usually the repercussion of motor-vehicle crashes and falls from height. Motor vehicle crashes are responsible for $48-68 \%$ of the pelvic injuries. These injuries often lead to lifetime disabilities and mortality ranges from $8.6 \%$ to up to $50 \%[17,4]$. According to [16] pelvic fractures account for $26 \%$ of the lower extremity trauma injuries, followed by tibia, fibula fractures and femur fractures. Pelvic injury can be accompanied by serious complications; solid evidence points to the fact that the high mortality rate is directly related to hemorrhage - either exsanguinations and intractable shock or complications of hemorrhage - infected pelvic hematomas and renal or multi-organ failure [2].

Detecting pelvic fracture is important in assessing the severity of an injury. Typically $\mathrm{x}$-ray images are used to diagnose a wide variety of pelvic injuries - asymmetry of the pelvis, destruction of the pelvis contour and widening of the of the pelvic ring. Computed Tomography (CT) of the pelvic regions is used to diagnose the more complicated cases, as it is more detailed. CT is usually used to confirm hip displacement, to detect acetabular fracture, to determine whether or not internal bleeding is present and to evaluate the severity of hemorrhage.

Based on collaborating physicians recommendation it was decided that identifying the edges of bones and the separation between them is much more important than capturing the core of the bone. While accurately identifying the edges of the bones can be used to determine the presence of fracture, knowledge about the inside of bone is not diagnostically significant.

Identifying and classifying bone by segmenting CT images is an important and challenging task in detecting pelvic fractures. The density of the outer bone - cortical bone - is much higher from that of the core - cancellous bone. Cortical bone appears to be bright and smooth while cancellous bone is darker and has a sponge like texture - therefore bone density cannot be uniformly characterized [12]. Differences in density can result in bone not being segmented correctly; regions of bone that need to be connected are identified as being separate. Another factor that adds to the complexity of the problem is the high number of slices in a CT scan. The differences between the positioning of the bones across the slices and the different types of fractures make identifying the presence of a fracture very difficult. Detecting the exact edges of bone is hard as they are diffused due to the partial volume effects in CT images. When distance between distinct bones is small, distinguishing between the bones becomes more difficult.

Pelvic fractures as well as open or closed internal bleeding can be diagnosed by trauma surgeons and radiologists after inspection of X-rays and CT scans of the pelvic region. Designing a system that would analyze these images and identify possible locations of fractures and/or bleeding will be very useful in improving the decision-making process. Typically a CT scan of the pelvic region has tens of slices. 
Designing an algorithm that would find possible problem areas in these slices could also reduce the time of decision making.

\subsection{Existing segmentation methods}

Grey level thresholding methods, although useful for important applications, may not be sufficient for bone segmentation in CT. Due to varying bone density the grey level of cancellous bone resembles that of the muscle tissue $[12,13]$. In addition, the small distance between bones, which makes the separation look brighter [12], and the partial volume effect, which diffuses the edges of bones, make the segmentation of neighboring bones more difficult.

Watershed segmentation is a region based approach that applies a gradient based segmentation technique. The gradient image is viewed as a height map and the image is segmented into different regions based on its topology; light pixels are considered high and dark pixels are low. However, the watershed algorithm can lead to oversegmentation, especially in noisy images such as bone CT $[12,10]$. Over-segmentation occurs because every local minimum in the image creates its own catchment basin. In marker-based watershed approaches, markers are used to reduce the local minima and to bound them in the region of interest in order to prevent over-segmentation [10].

Deformable models, such as Active Contour Models or Snakes, use closed parametric curves or surfaces that deform under internal and external forces. The closed curve must be placed near the desired boundary and allowed to iteratively relax. The advantages of standard deformable models include directly generating closed curves. The disadvantages of such algorithms are sensitivity to initialization and lack of tendency to converge to concave boundaries. Improved deformable model techniques apply implicit representation instead of explicit parameterization, and therefore are more topologically adaptable [3, 14].

The region competition methods combine the geometric features of snake models with the statistical properties of region growing. These methods segment the images by minimizing a generalized Minimum Description Length (MDL) criterion. The precision of boundary detection depends on the size of the sampling window, which in turn depends on the signal to noise ratio. The precision of the results depends on initialization - the choice of the initial seed points. [11]

In region growing methods an image is segmented by expanding a region starting with an initial seed point and iteratively adding neighboring pixels that satisfy certain similarity constraints. When a region stops growing another region is initialized with a seed that is not yet allocated into any region. The disadvantages of region growing methods include sensitivity to initialization - different seeds may lead to different results and seeds located on the edge complicate segmentation. Moreover, the growth process is dominated by the growth of the current region which might not be optimally initialized [9]. Seeded Region Growing (SRG) addresses these problems by ordering growth pixels according to a fitness criterion and creating competition between the growing regions [8]. Classic SRG depends on the order in which pixels in an image are processed. In Improved Seeded Region Growing (ISRG) algorithm this dependency is eliminated [1].

Level set methods (LSM) define a level-set (scalar) function over a regular grid and assume the initial curve is the zero-level set of the function. The curve is then deformed according to the solution given by a number of partial differential equations (PDEs) $[14,6,15]$. Different approaches on how the initial curve must evolve exist. Some approaches apply a global update of the level set function, while others use local update techniques. In the local approach the neighborhood of the zero-level set is windowed and only PDEs inside that neighborhood are solved to find the solution. [15]

Segmentation methods based on Gaussian Mixture Models (GMM) are parametric models that apply a mixture of Gaussians. A GMM based method of segmentation is usually initialized by a k-means segmentation and uses two GMMs; one to model the background and another to model the regions of interest [5].

In [13] a segmentation method is based on edge tracing. In the first instance, initial seeds are obtained either manually or by thresholding. Normal direction is used to determine the closest edge point to a certain seed and to correct the position of the seed if necessary. This is an iterative algorithm that stops when no seeds are left to examine. The method calculates normal direction for each seed, calculates a one dimensional signal based on the intensity of the pixels on the normal direction that is centered at the seed, detects the edge, identifies an edge point and estimates other possible edge points. As in other methods that use seed points for initialization, this method is sensitive to the choice of initial seeds. For this method, as for others that use seed points, initialization is important. Initial seeds need to be located fairly close to the desired edge. Post-processing is necessary, as in some cases false edges are detected. Normal direction is used in this case to delete the false edges that were created in the previous steps [13].

The rest of this paper is organized as follows. In Section 3 the step-by-step description of the algorithm is provided and the mathematical formulation of each step is presented. In Section 4 the segmentation results of the proposed method are compared with those of the GMM method. In Section 5 a short discussion on the capabilities and robustness of the proposed algorithm is provided. Finally, in Section 6 the conclusions are drawn. 


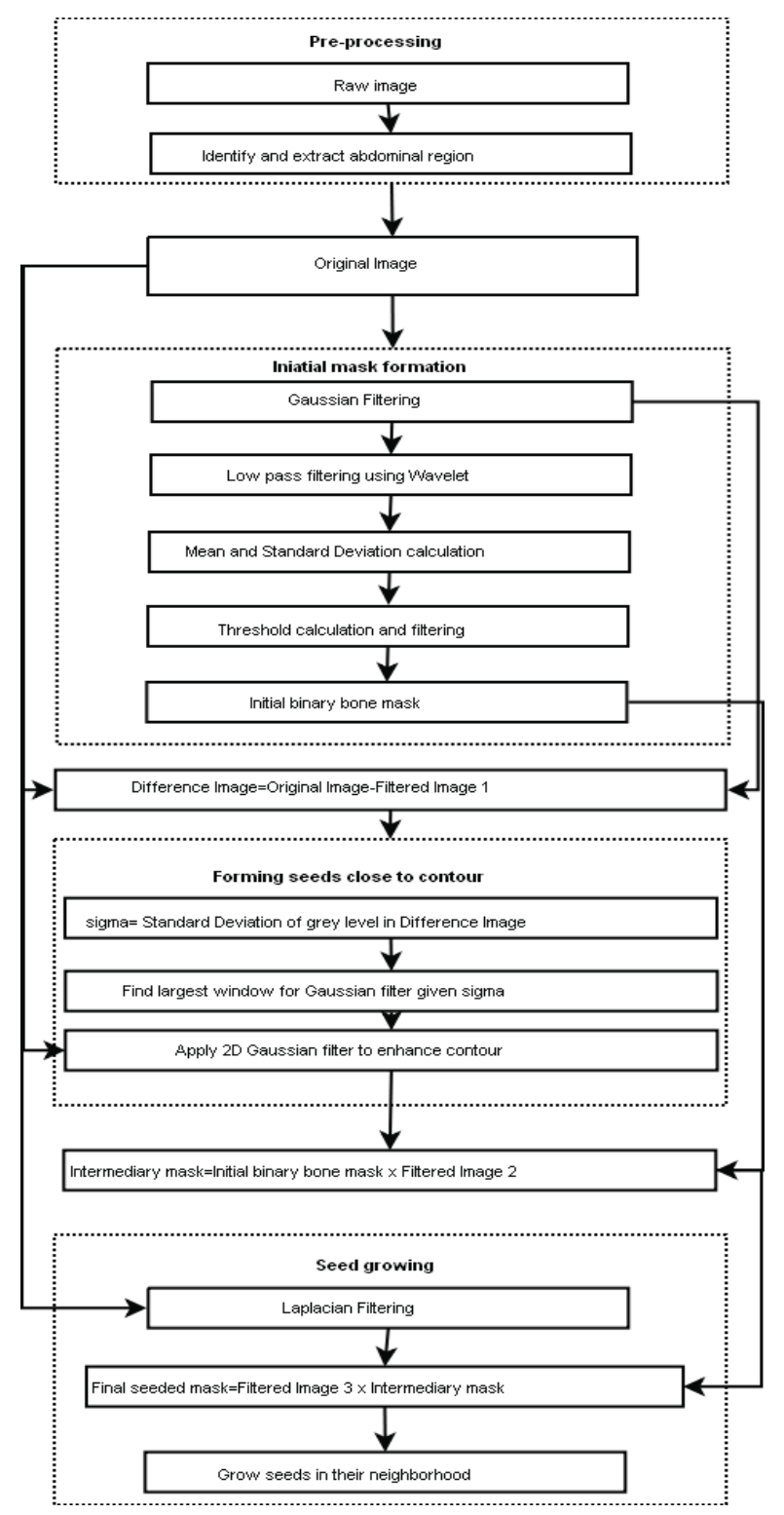

Figure 1. The diagram of the algorithm outline

\section{Method}

The method introduced by this paper is a systematic approach for segmentation of bone from the surrounding tissue. The schematic diagram of the algorithm is shown in Figure 1 and detailed in the following paragraphs.

\subsection{Pre-processing}

We proceed by developing a pre-processing method to distinguish between the abdominal region and the nearby artifacts that are irrelevant - CT table, cabling and lower extremities that are visible in some images. To accomplish the segmentation of abdomen from these objects that constitute the background the following pre-processing steps are implemented:

Step 1: Create a binary version of the raw image. All the pixels with grey level higher than zero become one. Pixels with grey value zero remain zero in the new image;

Step 2: Apply morphological operations to the binary image so that the different objects in the image are separated;

Step 3: Use blob analysis to select the object that has the largest area;

Step 4: Create a mask that is the size and shape of the object identified in Step 3.

\subsection{Initial mask formation}

Once the abdominal region is extracted and the background is removed from the image, the algorithm focuses on segmentation of bone. From now on the image of the abdomen will be referred to as "the original image".

As shown in Figure 1, the steps to obtain the initial segmentation are as follows:

Step 1: Apply a Gaussian filter to the original image;

Step 2: Apply Wavelet analysis to the resulting image and reconstruct the image using only the approximation matrix;

To obtain the initial bone mask, after applying a Gaussian filter to the original image and performing low pass filtering using Wavelet with Haar Transform on the resulting image. Assuming $G$ is the Gaussian filtered image and $W$ is the wavelet filtered image then:

$$
W=T \cdot G \cdot T^{T}
$$

where

$$
T=\frac{1}{\sqrt{2}}\left[\begin{array}{cc}
1 & 1 \\
1 & -1
\end{array}\right]
$$

Step 3: Calculate the means and deviation of the grey scale values that differ from zero, i.e. the pixels that are not background;

Step 4: Calculate threshold value as the sum of means and standard deviation of the grey scale values calculated at the previous step;

The threshold $t_{1}$ used is the sum between the modified means $m_{1}$ and standard deviation $s t_{1}$ of the grey values of the pixels that are not background: 


$$
t_{1}=m_{1}+s t_{1}
$$

where

$$
m_{1}=\frac{\sum_{x=0}^{N-1} \sum_{y=0}^{M-1} f(x, y)}{N \cdot M-\operatorname{Card}(S)}
$$

and

$$
\begin{gathered}
s t_{1}=\frac{\sqrt{\sum_{x=0}^{N-1} \sum_{y=0}^{M-1}\left[f(x, y)-m_{1}\right]^{2}}}{N \cdot M-\operatorname{Card}(S)} . \\
S=\{(x, y) \mid f(x, y)=0\}
\end{gathered}
$$

is the set of the pixels located in the background and that have zero grey level value. $\operatorname{Card}(S)$ is the notation used for the cardinality of set $S$.

Step 5: Create an initial binary mask for bone by thresholding the low pass image.

$$
\operatorname{mask}_{\text {initial }}(x, y)=\left\{\begin{array}{cc}
1 & \text {,if } \\
0 & \text {,otherwise }
\end{array} \quad f(x, y)>t_{1}\right.
$$

\subsection{Forming seeds close to contour}

After obtaining the initial binary mask for bone, the mask is refined and an intermediary binary mask is created. In order to further improve the initial mask, a two dimensional Gaussian filter will be applied to the original image. In the resulting image the contours will be enhanced and will be easier to identify. A short description of the steps taken in this stage is given below. Given the original image and the image after Gaussian filtering, the difference image between the two images is formed. Standard deviation in the grey level values of the difference image is calculated. Standard deviation, which will be referred to as sigma, is used to form the size of a filtering window. Specifically, based on the value of sigma we determine the most appropriate window size for the two dimensional Gaussian filter. In the process of obtaining the intermediary bone mask, a two dimensional Gaussian filter is applied to the original image. The resulting image $y_{g}$ grey values are given by:

$$
y_{g}(x, y)=\frac{1}{2 \pi \sigma^{2}} \cdot e^{-\frac{x^{2}+y^{2}}{2 \sigma^{2}}}
$$

After applying the Gaussian filter an intermediary bone mask is obtained through binary multiplication between the initial binary mask and the filtered original image.

$$
\operatorname{mask}_{\text {intermediary }}(x, y)=\operatorname{mask}_{\text {initial }}(x, y)-y_{g}(x, y)
$$

\subsection{Seed growing}

In the next step a Laplacian filter is applied to the original image and the result is multiplied with the intermediary bone mask. The seeded bone mask is obtained. Growing the seeds in the seeded bone mask in their neighborhood provides very good segmentation results. Region growing in the neighborhood of the seeds is accomplished as follows:

Step 1: For each seed in the seeded bone mask the neighbors in an $n x n$ neighborhood are identified (in this study $n=3$ );

Step 2: For each seed in the seeded bone mask the values of the gradient along the eight possible directions are calculated in the nxn neighborhood;

Step 3: For each of the neighbors in the $n x n$ neighborhood their $m x m$ neighborhood is identified (in this study $m=9$ );

Step 4: average grey level value of the neighbors in the $m x m$ neighborhood is calculated;

Step 5: a decision of adding a neighbor of a seed to the seeded bone mask is made based on two conditions:

a) the neighbor grey level value is greater than the average grey level value of the neighbors in the $m x m$ neighborhood;

b) the gradient value corresponding to the neighbor is greater or equal to -1 . This is a conservative criteria meant to keep the seeds from growing outside of the edges of the bone and minimizes the risk of separate bones being merged.

At this point a good segmentation of the bone tissue can be provided.

\section{Results}

Figures Figure 2 - Figure 5 illustrate some examples of segmentation results. In each of the four figures images labeled "1" are the raw images that are to be segmented. In images labeled "2" results of GMM segmentation can be observed, while images labeled " 3 " are the segmentation results created by the proposed method.

As mentioned before, accurately capturing bone edges is much more diagnostically important than conectivity in the core of bone.

Both algorithms provide accurate segmentation of the bone however, while GMM has a tendency to merge the bones when the distance between them is small. If small distances between bones affect the segmentation performance in this way then there will be a high risk of having the shattered pieces of bone from a fracture connected to each other in the segmented image. Our proposed method, on the other hand, is capable of distinguishing between separate bones even when the bones are in very close proximity. This aspect of the proposed method is extremely important 

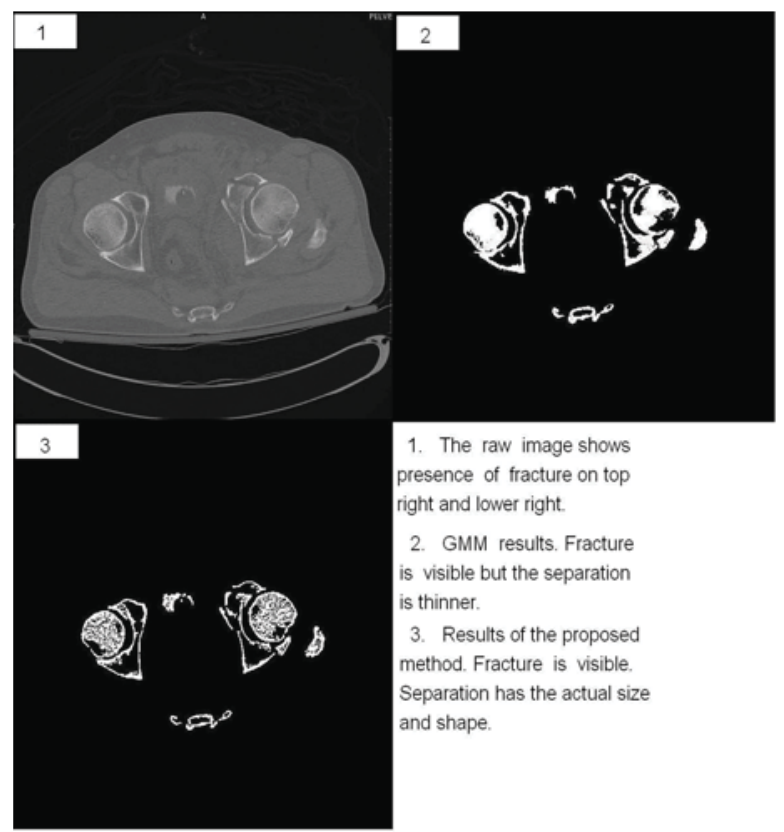

1. The raw image shows presence of fracture on top right and lower right.

2. GMM results. Fracture is visible but the separation is thinner.

3. Results of the proposed method. Fracture is visible. Separation has the actual size and shape.

Figure 2. Comparative results of the segmentation

as the segmentation will provide the grounds for identifying small fractures. For instance, in Figure 32 the fractured piece of bone in the right upper corner is fused with the round bone right next to it. This is not seen in the raw image Figure 31 . Comparatively, in Figure 33 which reflects the results of our segmentation method, the two bones are clearly segmented as separate pieces.

Another disadvantage of GMM segmentation of bone CT, compared to our proposed method, is that GMM tends to grow the bone regin to the regions representing other types of tissues when the bone edges are faint or blurred; this can be noticed in Figure 42 and Figure 52.

Also, it can be observed that in all images the bone contours for the proposed method are thinner, capturing the actual thickness of the original object. Images Figure 42 and Figure 43 present an example: on the right hand side of the image the bright line in Figure 4 2, representing GMM segmentation, is obviously thicker than in Figure 43 , showing our method which is very close to the actual image Figure 4 1.

\section{Discussion}

As it can be seen in Section 4, the results of the proposed segmentation method proved to be more accurate than those of some existing segmentation methods, such as GMM, for segmentation of bone CT images.

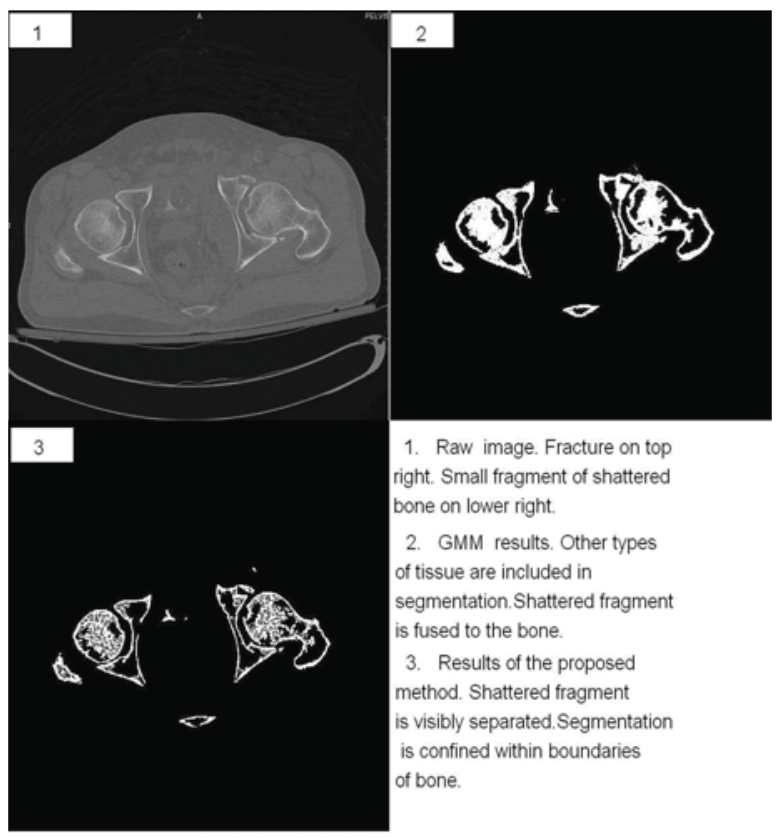

Figure 3. Comparative results of the segmentation

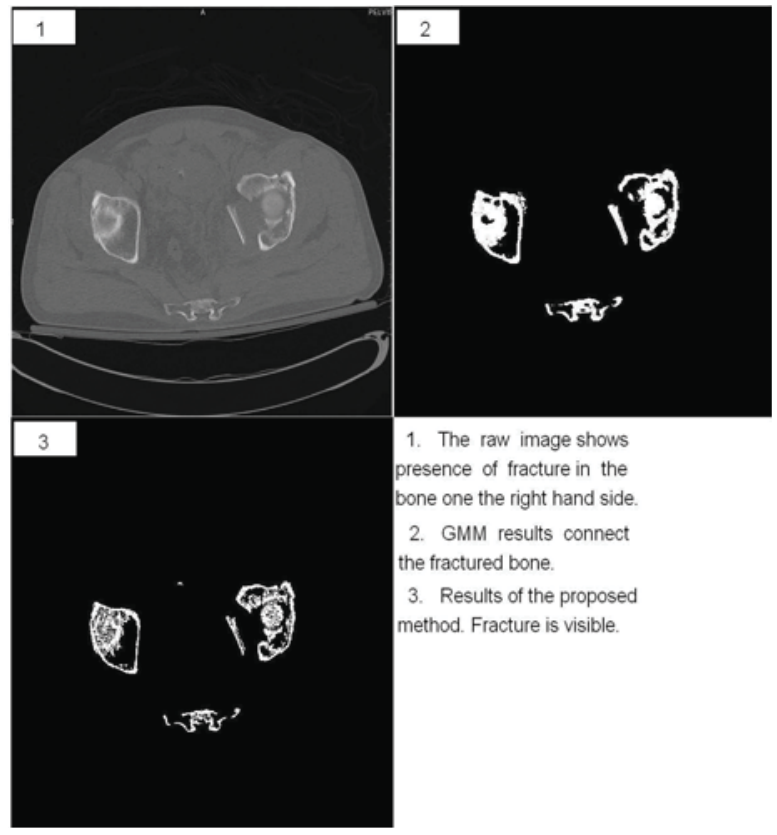

Figure 4. Comparative results of the segmentation 


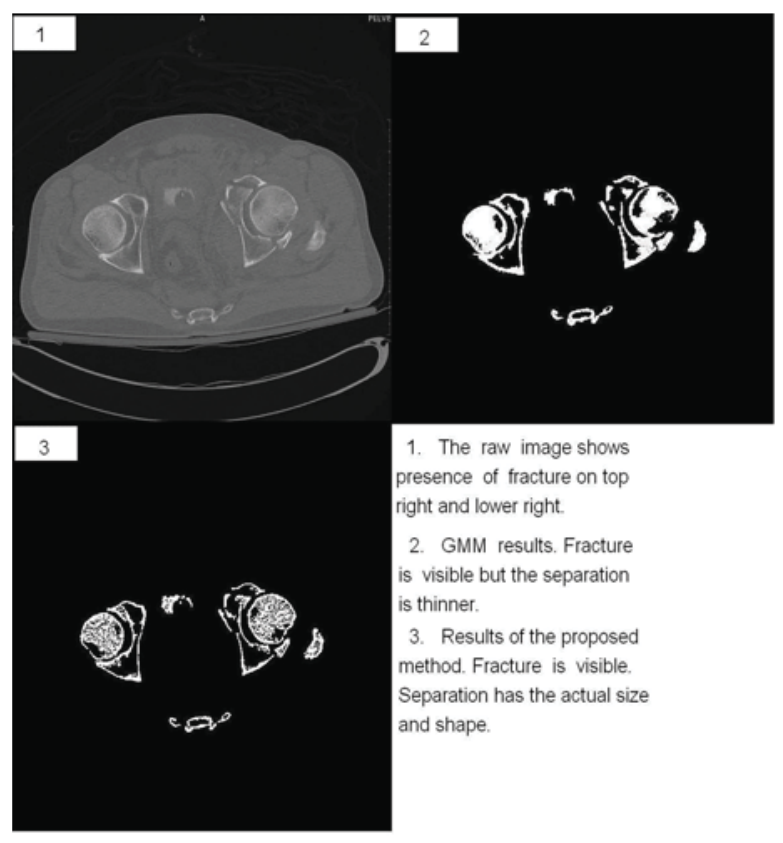

Figure 5. Comparative results of the segmentation

This automated bone segmentation method could help physicians in identifying the location of the fracture. The results provided by this method will be combined with physiological and demographic information to create an automated computer-aided decision making system.

The main advantages of our proposed bone CT segmentation method are:

1. While other segmentation methods have the tendency to grow bone regions to other regions representing other tissues when the bone edges are blurred, the proposed method confines itself inside the bone region while covering the entire bone tissue;

2. The proposed method does not merge separate bones even if the distance between them is small;

3 . The resulting lines in the segmented images are thinner in the proposed method, capturing actual contour of the original objects.

The algorithm, tested on approximately 300 images, successfully identifies bones both with sharp and faint edges.

Although the algorithm combines a number of methods and is highly hierarchical, the time complexity of the algorithm for the segmentation of typical CT images is significantly lower than methods such as GMM and Level-Sets.

Since segmentation of bone in CT images is an important step in creating an automated system. The proposed method can provide the grounds to create diagnostic recommendation for traumatic pelvic injuries.

\section{Conclusions and Future Work}

Accurate segmentation of CT pelvic images is a vital initial step in identifying fracture detection and ultimately creating an automated system to assist experts with diagnostic decisions on traumatic pelvic injuries. This modular computer-aided decision making system will combine fracture detection from $\mathrm{CT}$ and X-ray images with demographic and physiological data, to provide the best possible treatment recommendations and patient outcome predictions to physicians.

This paper presents a unified approach in segmentation of pelvic CT images that combines a suite of computational methods of segmentation that outperforms existing methods.

Future work includes detection of the location and severity of a fracture, identifying the presence of hemorrhage and correlating this information with the treatment outcomes so that accurate diagnostic recommendation can be provided to physicians. We are also planning to test this method on a larger database of patient CT images.

\section{Acknowledgement}

This material is based upon work supported by the National Science Foundation under Grant No.IIS0758410. The database used in this paper is courtesy of Carolinas Healthcare System.

\section{References}

[1] A. M. Authors and P. Jackway. An improved seeded region growing algorithm. Pattern Recognition Letters, 18(10):1065 - 1071, October 1997.

[2] B.-M. Y. Authors, D. M. Coldwell, J. W. R. Young, and A. R. Burgess. Hemorrhage associated with pelvic fractures: causes, diagnosis, and emergent management. American Journal of Roentgenology, 157(5):1005-1014, November 1991.

[3] D. L. P. Authors, C. Xu, and J. L. Prince. A survey of current methods in medical image segmentation. In Annual Review of Biomedical Engineering, January 1998.

[4] E. C. G. Authors, A. R. Burgess, S. M.-G. John H. Siegel, P. C. Dischinger, and S. M. Ho. Pelvic fracture mechanism of injury in vehicular trauma patients. The Journal of Trauma, 36(6):789-796, June 1994.

[5] H. G. Authors, A. Ruf, and J. Goldberger. Constrained gaussian mixture model framework for automatic segmentation of $\mathrm{mr}$ brain images. In IEEE Transactions on Medical Imaging, volume 25, pages 1233-1245, September 2006.

[6] H.-K. Z. Authors, S. Oshert, and R. Fedkiwt. Fast surface reconstruction using the level set method. In IEEE Workshop on Variational and Level Set Methods in Computer Vision, pages 194-201, 2001. 
[7] J. V. S. Authors, R. E. Broadhurst, S. M. Pizer, and E. L. Chaney. Regional Appearance in Deformable Model Segmentation. His Publisher, Erewhon, NC, 1999.

[8] R. A. Authors and L. Bischof. Seeded region growing. IEEE Transactions on Pattern Analysis and Machine Intelligence, 16(6):641-647, September 1994.

[9] R. C. G. Authors and R. E. Woods. Digital Image Processing. Pearson Prentice Hall, 3 edition, 2008.

[10] R. S. Authors, J. Alirezaie, and P. Babyn. Automatic lung segmentation in ct images using watershed transform. In IEEE International Conference on Image Processing, 2005.

[11] S. C. Z. Authors and A. Yuille. Region competition: Unifying snakes, region growing and bayes/mdl for multiband image segmentation. IEEE Transactions on Pattern Analysis and Machine Intelligence, 18(9):884-900, September 1996.

[12] T. B. S. Authors, H. Tek, J. J. Crisco, and B. B. Kimia. Segmentation of carpal bones from ct images using skeletally coupled deformable models. Medical Image Analysis, 7(1):21-45, March 2003.

[13] W. Y. Authors, P. Abolmaesumi, M. Greenspan, and R. E. Ellis. An estimation/correction algorithm for detecting bone edges in ct images. In IEEE Transactions on Medical Imaging, volume 24, pages 997-1010, August 2005.

[14] Y.-H. T. Authors and S. Osher. Level set methods in image science. In IEEE International Conference on Image Processing, volume 2, pages II 631 - II 634, September 2003.

[15] Y. S. Authors and W. C. Karl. A real-time algorithm for the approximation of level-set-based curve evolution. In IEEE Transactions on Image Processing, volume 17, pages 645656, May 2008.

[16] R. A. C. S. T. Center. Ciren program report. Technical report, University of Maryland National Study Center for Trauma and EMS, 2001.

[17] U. of Maryland National Study Center for Trauma/EMS Lower extremity injuries among restrained vehicle occupants. Technical report, University of Maryland National Study Center for Trauma/EMS, 2001. 